IJMMS 31:10 (2002) 627-633

PII. S0161171202109215

http://ijmms.hindawi.com

(c) Hindawi Publishing Corp.

\title{
THE MONAD INDUCED BY THE HOM-FUNCTOR IN THE CATEGORY OF TOPOLOGICAL SPACES AND ITS ASSOCIATED EILENBERG-MOORE ALGEBRAS
}

\author{
KOENA R. NAILANA
}

Received 15 September 2001

\begin{abstract}
We discuss the monad associated with the topology of pointwise convergence. We also
\end{abstract} study examples of the Eilenberg-Moore algebras for this monad.

2000 Mathematics Subject Classification: 18C15, 54B30, 54F05.

1. Introduction. Let Top denote the category of topological spaces and continuous functions. Let $\mathbb{R}$ denote the real line with the usual topology, and for each topological space $X$, let $C(X, \mathbb{R})$ be the set of continuous real-valued functions from $X$ to $\mathbb{R}$. Consider the contravariant hom-functor $C_{p}$ : Top $\rightarrow$ Top ${ }^{\text {op }}$ defined by assigning to each space $X$ the space of continuous real-valued functions with the topology of pointwise convergence. We denote this space by $C_{p} X$. The space $C_{p} X$ has been extensively studied. A fundamental reference on $C_{p} X$ is Arkhangel'skii [2]. We recall that the subbasic open sets of $C_{p} X$ are sets of the form $[f, V]$, where $[x, V]=\left\{f \in C_{p} X: f(x) \in\right.$ $V, V$ open in $\mathbb{R}\}$.

2. The monad induced by the hom-functor in Top and the associated $M$-algebras. We now consider the composite functor $C_{p}^{\mathrm{op}} C_{p}$ : Top $\rightarrow$ Top ${ }^{\text {op }} \rightarrow$ Top where $C_{p}^{\mathrm{op}}$ is the dual functor. Let $M=C_{p}^{\mathrm{op}} C_{p}$. If $x \in X$, then the function $\hat{x}: C_{p} X \rightarrow \mathbb{R}$ defined by $\hat{x}(f)=$ $f(x)$ is called the evaluation map at $x$. The following propositions are important since they ensure that our morphisms are continuous. The proofs are straightforward and will be omitted.

Proposition 2.1. (i) For all $x \in X, \hat{x}: C_{p} X \rightarrow \mathbb{R}$ is continuous.

(ii) For all $g \in C_{p} X, \hat{g}: M C_{p} X \rightarrow \mathbb{R}$ is continuous.

Proposition 2.2. Let $X$ be any topological space. Then

(i) $\eta_{X}: X \rightarrow M X$, where $\eta_{X}(x)=\hat{x}$ is continuous.

(ii) $\mu_{X}: M M X \rightarrow M X$, where $\mu_{X}(\gamma)[g]=\gamma(\hat{g})$ is continuous.

We recall from [1] that a monad on a category $\mathbb{A}$ is a triplet $\mathbb{M}=(M, \eta, \mu)$ consisting of a functor $M: \mathbb{A} \rightarrow \mathbb{A}$ and natural transformations $\eta: \operatorname{id}_{\mathbb{A}} \rightarrow M$ and $\mu: M M \rightarrow M$ such that $\mu \circ M \mu=\mu \circ \mu M, \mu \circ M \eta=\mathrm{id}$, and $\mu \circ \eta M=\mathrm{id}$.

Proposition 2.3. The triplet $(M, \eta, \mu)$, where $\eta: \mathrm{id}_{\mathrm{Top}} \rightarrow M$ and $\mu: M M \rightarrow M$ are defined by $\eta_{X}(x)=\hat{x}$ and $\mu_{X}(\gamma)[g]=\gamma(\hat{g})$, respectively, where $x \in X, g \in C_{p} X$, is $a$ monad. 
Proof. We first check that $\eta: \operatorname{id}_{\text {Top }} \rightarrow M$ and $\mu: M M \rightarrow M$ are natural transformations. Let $f: X \rightarrow Y$ be a continuous function. We show that $M(f) \circ \eta_{X}=\eta_{Y} \circ f$. We define $\eta_{X}$ and $M(f)$ by $\eta_{X}(x)=\hat{x}$ and $M(f)(\gamma)[g]=\gamma(g \circ f)$ where $g: Y \rightarrow \mathbb{R}$ is continuous, $\gamma \in M(X)$, and ${ }^{\wedge}$ denotes evaluation, for example, $\hat{x}(g)=g(x)$. Then $M(f) \circ \eta_{X}(x)=M(f)(\hat{x})$. Let $g \in C_{p} X$. Then $M(f)(\hat{x})[g]=\hat{x}(g \circ f)=g \circ f(x)=$ $g(f(x))=\widehat{f(x)}[g]$. Hence $M(f)(\hat{x})=\widehat{f(x)}$. Now $\eta_{Y} \circ f(x)=\eta_{Y}(f(x))=\widehat{f(x)}$. Let $g \in C_{p} X$. Then $\widehat{f(x)}[g]=g(f(x))$. Hence $M(f) \circ \eta_{X}=\eta_{Y} \circ f$. We define $\mu_{X}$ by $\mu_{X}(\gamma)[g]=\gamma(\hat{g})$ where $\gamma \in M M(X), g \in C_{p} X$, and $\hat{g}$ denotes the evaluation function at $g$, that is, $\hat{g}: M(X) \rightarrow \mathbb{R}$. We now show that $\mu: M M \rightarrow M$ is a natural transformation, that is, $M(f) \circ \mu_{X}=\mu_{Y} \circ M^{2}(f)$. Let $h \in C_{p} Y$. Then

$$
\left(M(f) \circ \mu_{X}\right)(\gamma)[h]=M(f)\left(\mu_{X}(\gamma)\right)[h]=\mu_{X}(\gamma)(h \circ f)=\gamma(\widehat{h \circ f}) .
$$

On the other hand,

$$
\begin{aligned}
\mu_{Y} \circ M^{2}(f)(\gamma)[h] & =\mu_{Y}\left(M^{2}(f)(\gamma)\right)[h] \\
& =M^{2}(f)(\gamma)(\hat{h}) \\
& =M(M(f))(\gamma)(\hat{h}) \\
& =\gamma(\hat{h} \circ M(f)) .
\end{aligned}
$$

Let $\lambda: C_{p} X \rightarrow \mathbb{R}$. Then $(\hat{h} \circ M(f))(\lambda)=\hat{h}(M(f)(\lambda))=M(f)(\lambda)[h]=\lambda(h \circ f)=$ $\widehat{h \circ f}(\lambda)$. Therefore, $\hat{h} \circ M(f)=\widehat{h \circ f}$. From the equations

$$
\begin{aligned}
M(f) \circ \mu_{X}(\gamma)[h] & =\gamma(\widehat{h \circ f}), \\
\mu_{Y} \circ M^{2}(f)(\gamma)[h] & =\gamma(\hat{h} \circ M(f)), \\
(\hat{h} \circ M(f))(\lambda) & =\widehat{h \circ f}(\lambda),
\end{aligned}
$$

we get $M(f) \circ \mu_{X}=\mu_{Y} \circ M^{2}(f)$. Therefore $\mu: M M \rightarrow M$ is a natural transformation. We now show that the other monad conditions are satisfied. First, we show that $\mu_{X} \circ M \eta=$ id. We prove that $\mu_{X} \circ M \eta=$ id and $\mu_{X} \circ \eta_{M}=$ id. Let $\gamma \in M(X)$ and $f \in C_{p} X$. Then $\hat{f}: M(X) \rightarrow \mathbb{R}$ and $\left(\mu_{X} \circ M \eta\right)(\gamma) \in M(X)$. Then $\left(\mu_{X} \circ M \eta\right)(\gamma)[f]=$ $\mu_{X}(M \eta(\gamma))[f]=M \eta(\gamma)(\hat{f})=\gamma(\hat{f} \circ \eta)=\gamma[f]$. Therefore $\mu_{X} \circ M \eta=$ id. On the other hand $\left(\mu_{X} \circ \eta_{M}\right)(\gamma)[f]=\mu_{X}\left(\eta_{M}\right)(\gamma)[f]=\eta_{M}(\gamma)[\hat{f}]=\hat{\gamma}(\hat{f})=\hat{f}(\gamma)=\gamma(f)$. Therefore $\mu_{X} \circ \eta_{M}=$ id. Second, we show that $\mu_{X} \circ \mu_{M}=\mu_{X} \circ M \mu_{X}$. We prove that $\mu_{X} \circ \mu_{M}=$ $\mu_{X} \circ M \mu_{X}$. Let $\gamma \in M M M(X)$. Then $\mu_{X} \circ M \mu_{X}(\gamma) \in M(X)$. Let $f \in C_{p} X$. Then $\left(\mu_{X} \circ\right.$ $\left.M \mu_{X}\right)(\gamma)[f]=\mu_{X}\left(M \mu_{X}(\gamma)\right)[f]=M \mu_{X}(\gamma)[\hat{f}]=\gamma\left(\hat{f} \circ \mu_{X}\right)=\gamma(\hat{\hat{f}})$. On the other hand, $\left(\mu_{X} \circ \mu_{M}\right)(\gamma)[f]=\mu_{X}\left(\mu_{M}(\gamma)\right)[f]=\mu_{M}(\gamma)(\hat{f})=\gamma(\hat{\hat{f}})$. Therefore $\mu_{X} \circ \mu_{M}=\mu_{X} \circ M \mu_{X}$. Therefore $(M, \eta, \mu)$ is a monad.

If $\mathbb{M}=(M, \eta, \mu)$ is a monad on $\mathbb{A}$, then $\left(A, h_{A}\right)$ is called an Eilenberg-Moore algebra or simply an $M$-algebra if the algebra map $h_{A}: M A \rightarrow A$ satisfies $h_{A} \circ \eta_{A}=\mathrm{id}_{A}$ and $h_{A} \circ M h_{A}=h_{A} \circ \mu_{A}$.

We now look at examples of the $M$-algebras of the monad $(M, \eta, \mu)$. 
Proposition 2.4. The real line $\mathbb{R}$ is an M-algebra.

Proof. We define $h_{\mathbb{R}}: M \mathbb{R} \rightarrow \mathbb{R}$ as $\hat{\mathbf{1}}_{\mathbb{R}}$, that is, the identity map with respect to $\mathbb{R}$, and show that the $M$-algebra conditions are satisfied. It is obvious that the map $h_{\mathbb{R}}$ is continuous. Let $x \in \mathbb{R}$. Then $h_{\mathbb{R}} \circ \eta_{\mathbb{R}}(x)=h_{\mathbb{R}}(\hat{x})=\hat{x}\left(\mathbf{1}_{\mathbb{R}}\right)=\mathbf{1}_{\mathbb{R}}(x)$. Therefore $h_{\mathbb{R}} \circ \eta_{\mathbb{R}}=\mathbf{1}_{\mathbb{R}}$. Now let $\gamma \in M M(\mathbb{R})$. Then $h_{\mathbb{R}} \circ \mu_{\mathbb{R}}(\gamma)=\hat{\mathbf{1}}_{\mathbb{R}}\left(\mu_{\mathbb{R}}(\gamma)\right)=\mu_{\mathbb{R}}(\gamma)\left(\mathbf{1}_{\mathbb{R}}\right)=\gamma\left(\hat{\mathbf{1}}_{\mathbb{R}}\right)$. On the other hand, $h_{\mathbb{R}} \circ M h_{\mathbb{R}}(\gamma)=\hat{\mathbf{1}}_{\mathbb{R}}\left(M \hat{\mathbf{1}}_{\mathbb{R}}(\gamma)\right)=\hat{\mathbf{1}}_{\mathbb{R}}\left(\gamma \circ C_{p}\left(\hat{\mathbf{1}}_{\mathbb{R}}\right)\right)=\gamma \circ C_{p}\left(\hat{\mathbf{1}}_{\mathbb{R}}\right)\left(\mathbf{1}_{\mathbb{R}}\right)=$ $\gamma\left(C_{p}\left(\hat{\mathbf{1}}_{\mathbb{R}}\right)\left(\mathbf{1}_{\mathbb{R}}\right)\right)=\gamma\left(\mathbf{1}_{\mathbb{R}} \circ \hat{\mathbf{1}}_{\mathbb{R}}\right)=\gamma\left(\hat{\mathbf{1}}_{\mathbb{R}}\right)$. Therefore $h_{\mathbb{R}} \circ \mu_{\mathbb{R}}=h_{\mathbb{R}} \circ M h_{\mathbb{R}}$.

Proposition 2.5. For each $X \in \mathrm{Top}, C_{p} X$ is an $M$-algebra with $h_{C_{p} X}=C_{p}\left(\eta_{X}\right)$.

Proof. We first define $h_{C_{p} X}: M C_{p} X \rightarrow C_{p} X$. Let $\varphi \in M C_{p} X$. We define $h_{C_{p} X}$ by $h_{C_{p} X}(\varphi)=\varphi \circ \eta_{X}=C_{p} \eta_{X}(\varphi)$. Then the map $h_{C_{p} X}$ is continuous, since it is the composite of continuous functions $\varphi$ and $\eta_{X}$. We now show that the conditions for an $M$-algebra are satisfied. Thus, we must show that $h_{C_{p} X} \circ \eta_{C_{p} X}=\operatorname{id}_{C_{p X}}$. Let $f \in C_{p} X$. Then $h_{C_{p} X} \circ \eta_{C_{p} X}(f)=h_{C_{p} X}\left(\eta_{C_{p} X}(f)\right)=C_{p} \eta_{X}(\hat{f})=\hat{f} \circ \eta_{X}=f=\operatorname{id}_{C_{p} X}(f)$, since $\hat{f} \circ \eta_{X}(x)=\hat{f}\left(\eta_{X}(x)\right)=\hat{x}(f)=f(x)$. Therefore $h_{C_{p} X} \circ \eta_{C_{p} X}=\operatorname{id}_{C_{p} X}$.

We must now show that $h_{C_{p} X} \circ \mu_{C_{p} X}=h_{C_{p} X} \circ M h_{C_{p} X}$. Let $\gamma \in M M C_{p} X$. Then $h_{C_{p} X} \circ$ $\mu_{C_{p} X}(\gamma)=h_{C_{p} X}\left(\mu_{C_{p} X}(\gamma)\right)=C_{p} \eta_{X}\left(\mu_{C_{p} X}(\gamma)\right)=\mu_{C_{p} X}(\gamma) \circ \eta_{X}$. Now let $x \in X$. Then $\mu_{C_{p} X}(\gamma) \circ \eta_{X}(x)=\mu_{C_{p} X}(\gamma)(\hat{x})=\gamma(\hat{\hat{x}})$. On the other hand, $h_{C_{p} X} \circ M h_{C_{p} X}(\gamma)=C_{p} \eta_{X} \circ$ $M C_{p} \eta_{X}(\gamma)=C_{p}\left(M \eta_{X} \circ \eta_{X}\right)(\gamma)=\gamma \circ M \eta_{X} \circ \eta_{X}$. Let $x \in X$. Then $M \eta_{X} \circ \eta_{X}(x)=M \eta_{X}(\hat{x})$ $=\hat{x} \circ C_{p} \eta_{X}=\hat{\hat{x}}$. Therefore $h_{C_{p} X} \circ \mu_{C_{p} X}=h_{C_{p} X} \circ M h_{C_{p} X}$. Hence $C_{p} X$ is an $M$-algebra.

Proposition 2.6. Retracts of $C_{p} X$ are $M$-algebras.

Proof. Let $g: C_{p} Y \rightarrow X$ be a retraction. Then there is a continuous function $f$ : $X \rightarrow C_{p} Y$ such that $g \circ f=\operatorname{id}_{X}$. The following diagram will help us define the algebra map $h_{X}: M X \rightarrow X:$

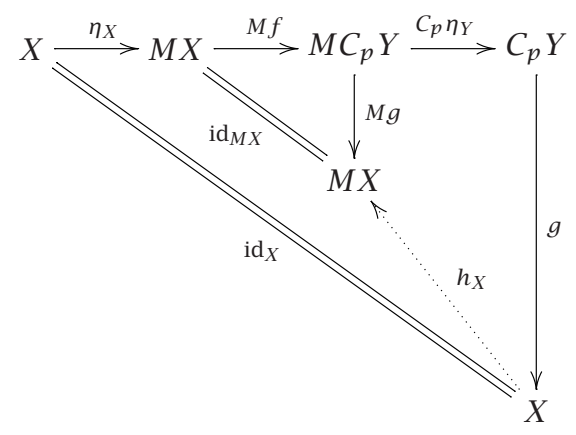

Define

$$
h_{X}=g \circ C_{p} \eta \circ M f=g \circ C_{p}\left(C_{p} f \circ \eta_{Y}\right) .
$$

Since $h_{X}$ is the composite of continuous functions, then it is continuous. 
Now,

$$
\begin{aligned}
h_{X} \circ \eta_{X}(x) & =h_{X}(\hat{x})=g\left(C\left(C f \circ \eta_{Y}\right)(\hat{x})\right)=g\left(\hat{x} \circ C f \circ \eta_{Y}\right) \\
& =g\left(\widehat{f(x)} \circ \eta_{Y}\right)=g(f(x))=\operatorname{id}_{X}(x),
\end{aligned}
$$

since $g$ is a retraction.

We now show that $h_{X} \circ \mu_{X}=h_{X} \circ M h_{X}$. Let $\gamma \in M M X$. Then

$$
\begin{aligned}
h_{X} \circ \mu_{X}(\gamma) & =h_{X}\left(\mu_{X}(\gamma)\right)=g \circ C_{p}\left(C_{p} f \circ \eta_{Y}\right)\left(\mu_{X}(\gamma)\right) \\
& =g\left(C_{p}\left(C_{p} f \circ \eta_{Y}\right)\left(\mu_{X}(\gamma)\right)\right)=g\left(\mu_{X}(\gamma) \circ C_{p} f \circ \eta_{Y}\right) .
\end{aligned}
$$

If $k \in C_{p} X$, then

$$
\mu_{X}(\gamma)(k)=\gamma(\hat{k})
$$

On the other hand,

$$
\begin{aligned}
h_{X} \circ M h_{X} & =g \circ C_{p}\left(C_{p} f \circ \eta_{Y}\right) \circ M\left(g \circ C_{p}\left(C_{p} f \circ \eta_{Y}\right)\right) \\
& =g \circ C_{p} \eta_{Y} \circ M f \circ M g \circ M C_{p}\left(C_{p} f \circ \eta_{Y}\right) \\
& =g \circ C_{p} \eta_{Y} \circ M(f \circ g) \circ M C_{p}\left(C_{p} f \circ \eta_{Y}\right) \\
& =g \circ C_{p} \eta_{Y} \circ M\left(\operatorname{id}_{X}\right) \circ M C_{p}\left(C_{p} f \circ \eta_{Y}\right) \\
& =g \circ C_{p} \eta_{Y} \circ M C_{p}\left(C_{p} f \circ \eta_{Y}\right) \\
& =g \circ C_{p}\left(M\left(C_{p} f \circ \eta_{Y}\right) \circ \eta_{Y}\right) \\
& =g \circ C_{p}\left(\eta_{C_{p}} \circ C_{p} f \circ \eta_{Y}\right) .
\end{aligned}
$$

Now,

$$
\begin{aligned}
h_{X} \circ M h_{X}(\gamma) & =g\left(C_{p}\left(\eta_{C_{p} X} \circ C_{p} f \circ \eta_{Y}\right)(\gamma)\right) \\
& =g\left(\gamma \circ \eta_{C_{p} X} \circ C_{p} f \circ \eta_{Y}\right) .
\end{aligned}
$$

We only need to show that $\gamma \circ \eta_{C_{p} X}=\mu_{X}$. Let $k \in C_{p} X$. Then $\gamma \circ \eta_{C_{p} X}(k)=\gamma\left(\eta_{C_{p} X}(k)\right)$ $=\gamma(\hat{k})$. From (2.8), we have $\gamma \circ \eta_{C_{p} X}=\mu_{X}$ and therefore $h_{X} \circ \mu_{X}=h_{X} \circ M h_{X}$. Hence retracts of $C_{p} X$ are $M$-algebras.

3. The algebra morphisms and the transfer of ring structure from $M X$ to $X$ for an $M$-algebra $\left(X, h_{X}\right)$. For an $M$-algebra $\left(X, h_{X}\right)$ the ring structure on $M X$ can be transferred to $X$, via $h_{X}$, in such a way that $X$ becomes a ring with respect to the induced operations.

DEFINITION 3.1. On an $M$-algebra $\left(X, h_{X}\right)$ define

(i) $x_{1}+x_{2}$ to be $h_{X}\left(\eta_{X}\left(x_{1}\right)+\eta_{X}\left(x_{2}\right)\right)$,

(ii) $x_{1} \cdot x_{2}$ to be $h_{X}\left(\eta_{X}\left(x_{1}\right) \cdot \eta_{X}\left(x_{2}\right)\right)$.

In addition to the ring structure defined above we also define the scalar multiplication in the following way: define $t x$ to be $h_{X}\left(t \eta_{X}(x)\right)$, where $t$ is a scalar.

According to Definition 3.1, $C_{p} X$ (being an $M$-algebra, Proposition 2.5) has now two concepts of the operations "+" and ".", the natural one defined pointwise

$$
\left(h_{X}(x+y)=h_{X}(x)+h_{X}(y), h_{X}(x y)=h_{x}(x) h_{X}(y)\right)
$$


and Definition 3.1. The same applies to $M X$. We omit the straightforward proof of the following proposition.

Proposition 3.2. The natural operations on $M X$ defined pointwise coincide with the corresponding ones defined above.

LEMMA 3.3. The topology on $X$ is initial with respect to $\eta_{X}$, that is, $X$ has the weak topology induced by $\eta_{X}$ into $C_{p} C_{p} X=M X$.

Proof. Basic neighborhoods of $\eta_{X}(x)$ have inverse images of $\eta_{X}$ of the form $\cap_{i=1}^{n} f_{i}^{-1}\left[W_{i}\right]$.

LEMMA 3.4 [2]. Let $\varphi \in C_{p} C_{p} X$ such that $\varphi:\left(C_{p} X, h_{C_{p} X}\right) \rightarrow\left(\mathbb{R}, h_{\mathbb{R}}\right)$ is a linear functional. Then there are $x_{1}, \ldots, x_{n} \in X, \lambda_{1}, \ldots, \lambda_{n} \in \mathbb{R}$ such that $\varphi=\sum_{i=1}^{n} \lambda_{i} \hat{x}_{i}$.

Proposition 3.5. If $\varphi:\left(C_{p} X, h_{C_{p} X}\right) \rightarrow\left(\mathbb{R}, h_{\mathbb{R}}\right)$ is a nontrivial continuous multiplicative linear functional, then there is $x \in X$ such that $\varphi=\hat{x}$, that is, $\varphi$ is a point evaluation.

Proof. By Lemma 3.4, there are points $x_{1}, \ldots, x_{n} \in X$, and scalars $\lambda_{1}, \ldots, \lambda_{n} \in \mathbb{R}$ such that $\varphi=\sum_{i=1}^{n} \lambda_{i} \hat{x}_{i}$ where $\lambda_{i}=\varphi\left(g_{i}\right), g_{i} \in C_{p} X$ being such that $g_{i}\left(x_{i}\right)=1$, $g_{i}\left(x_{j}\right)=0$ for $i \neq j, 0 \leq g_{i} \leq 1$. Now $\varphi\left(g_{k}^{2}\right)=\varphi\left(g_{k}\right)^{2}=\lambda_{k}$. Also $\varphi\left(g_{k}^{2}\right)=\sum_{i=1}^{n} \lambda_{i} \hat{x}_{i}\left(g_{k}^{2}\right)$ $=\sum_{i=1}^{n} \lambda_{i} g_{k}^{2}\left(x_{i}\right)=\lambda_{k}$.

Thus $\lambda_{k}=\lambda_{k}^{2}$, so that $\lambda_{k}=0$ or $\lambda_{k}=1$ for $k=1,2, \ldots, n$. Moreover, $\lambda_{k}=g_{k}\left(x_{k}\right) \geq 0$. Furthermore, $\varphi(\mathbf{1})=1$ gives $1=\varphi(\mathbf{1})=\sum_{i=1}^{n} \lambda_{i} \hat{x}_{i}(\mathbf{1})=\sum_{i=1}^{n} \lambda_{i}$. Consequently, all $\lambda_{i}$ 's except one are zero, the exceptional one being one 1 . Let $x=x_{l}$, where $\lambda_{l}=1$. Then $\lambda_{i}=0$ for $i \neq l$, so that $\varphi=\lambda_{l} \hat{x}_{l}=\hat{x}_{l}$.

Proposition 3.6. Let $\varphi:\left(C_{p} X, h_{C_{p} X}\right) \rightarrow\left(\mathbb{R}, h_{\mathbb{R}}\right)$ be an algebra map. Then $\varphi$ is a continuous ring homomorphism.

Proof. Given $f, g \in C_{p} X$, consider $\eta_{C_{p} X}(f)+\eta_{C_{p} X}(g)$ in $M C_{p} X$. We have

$$
h_{\mathbb{R}} \circ C^{2} \varphi\left(\eta_{C_{p} X}(f)+\eta_{C_{p} X}(g)\right)=h_{\mathbb{R}} \circ M \varphi\left(\eta_{C_{p} X}(f)\right)+h_{\mathbb{R}} \circ M \varphi\left(\eta_{C_{p} X}(g)\right)
$$

by Lemma 3.4.

Hence $\varphi \circ h_{X}\left(\eta_{C_{p} X}(f)+\eta_{C_{p} X}(g)\right)=\varphi \circ h_{X}\left(\eta_{C_{p} X}(f)\right)+\varphi \circ h_{X}\left(\eta_{C_{p} X}(g)\right)$, so that $\varphi(f+g)=\varphi(f)+\varphi(g)$, since $h_{X}$ preserves the ring structure. Similarly, $\varphi(f \cdot g)=$ $\varphi(f) \cdot \varphi(g)$. We also have $\varphi(t f)=t \varphi(f), t \in \mathbb{R}$. Moreover $\varphi(\mathbf{1})=1$, where $\mathbf{1}$ denotes the constant function with value equal to 1 .

Proposition 3.7. Every algebra map $\varphi:\left(C_{p} X, h_{C_{p} X}\right) \rightarrow\left(\mathbb{R}, h_{\mathbb{R}}\right)$ is a point evaluation map.

Proof. By the above proposition, $\varphi$ is a continuous ring homomorphism, that is, a continuous multiplicative linear functional. Thus, there is some $x \in X$ such that $\varphi(f)=f(x)$ for all $f$ in $C_{p} X$, by the above proposition.

THEOREM 3.8. The algebra morphisms $\varphi:\left(C_{p} X, h_{C_{p} X}\right) \rightarrow\left(\mathbb{R}, h_{\mathbb{R}}\right)$ are precisely the morphisms $\hat{x}$, where $x \in X$, that is, the point evaluation map. 
Proof. Suppose $\varphi=\hat{x}$, for some $x \in X$. Let $\gamma \in M C_{p} X$. Take $h_{X}=C_{p} \eta_{C_{p} X}$ and $h_{\mathbb{R}}=\hat{1}_{\mathbb{R}}$. Then $\varphi \circ h_{X}(\gamma)=\hat{x} \circ C_{p} \eta_{C_{p} X}(\gamma)=\hat{x}\left(C_{p} \eta_{C_{p} X}(\gamma)\right)=\gamma \circ \eta_{X}(x)=\gamma(\hat{x})$. On the other hand, $h_{\mathbb{R}} \circ M(\varphi)(\gamma)=h_{\mathbb{R}}(M(\varphi)(\gamma))=\hat{1}_{\mathbb{R}}(M(\varphi)(\gamma))=M(\varphi)(\gamma)\left(\mathbf{1}_{\mathbb{R}}\right)=\gamma\left(\mathbf{1}_{\mathbb{R}} \circ\right.$ $\varphi)=\gamma(\varphi)=\gamma(\hat{x})$. Therefore, $\varphi \circ h_{X}=h_{\mathbb{R}} \circ M(\varphi)$ and thus $\varphi=\hat{x}$ is an algebra morphism. The converse follows from Proposition 3.7.

Proposition 3.9. The algebra morphisms $\varphi:\left(C_{p} X, h_{C_{p} X}\right) \rightarrow\left(C_{p} Y, h_{C_{p} Y}\right)$ are the maps $C_{p}(f)$, where $f: Y \rightarrow X$ is continuous.

PROoF. Suppose that $\varphi:\left(C_{p} X, h_{C_{p} X}\right) \rightarrow\left(C_{p} Y, h_{C_{p} Y}\right)$ is an algebra map. Given $y \in$ $Y, \hat{y} \circ \varphi:\left(C_{p} X, h_{C_{p} X}\right) \rightarrow\left(\mathbb{R}, h_{\mathbb{R}}\right)$ is an algebra map, since the composition of two algebra maps is an algebra map. Thus the following diagram is commutative:

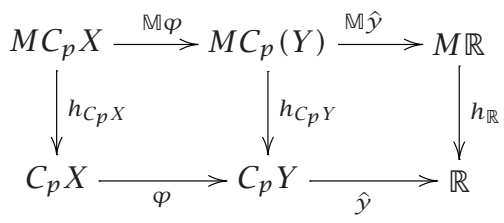

By Theorem 3.8, $\hat{y} \circ \varphi=\hat{x}$ for some $x \in X$. Put $x=f(y)$. Thus $f$ maps $Y$ into $X$. Since $X$ has the initial topology induced by $\eta_{X}, f$ will be continuous if $\eta_{X} \circ f$ is continuous. Now $\eta_{X} \circ f(y)=\hat{x}=\hat{y} \circ \varphi=C \varphi\left(\eta_{Y}(y)\right)$. Thus $\eta_{X} \circ f=C \varphi \circ \eta_{Y}$, so that $\eta_{X} \circ f$ is continuous, hence $f$ is continuous, as required. It remains to prove that $\varphi=C f$. Since the functions $\hat{y}$ distinguish the points of $C_{p} Y$, it suffices to prove that $\hat{y} \circ \varphi=\hat{y} \circ C f$ for every $y \in Y$. Now $\hat{y}(C f(g))=\hat{y}(g \circ f)=g \circ f(y)=g(f(y))=$ $g(x)$. Also $\hat{y}(\varphi(g))=\hat{y} \circ \varphi(g)=\hat{x}(g)=g(x)$. Hence $\hat{y} \circ \varphi=\hat{y} \circ C f$ for all $y \in Y$, so that $\varphi=C f$.

Conversely suppose the morphism $\varphi:\left(C_{p} X, h_{C_{p} X}\right) \rightarrow\left(C_{p} Y, h_{C_{p} Y}\right)$ is such that $\varphi=$ $C f$. Then by Proposition 3.9, $\varphi$ is an algebra morphism.

Proposition 3.10. The map $h_{C_{p} X}: M C_{p} X \rightarrow C_{p} X$ preserves the ring structure of the function spaces, operations being defined pointwise.

Proof. Let $\varphi, \psi \in M C_{p} X$, so that $\varphi, \psi: M X \rightarrow \mathbb{R}$. The maps $\varphi+\psi, \varphi \cdot \psi$, and $t \varphi$ (where $t \in \mathbb{R}$ ) are both defined pointwise, so that $(\varphi+\psi)(\lambda)=\varphi(\lambda)+\psi(\lambda), \varphi \cdot \psi(\lambda)=$ $\varphi(\lambda) \cdot \psi(\lambda)$, and $(t \varphi)(\lambda)=t \varphi(\lambda)$, for all $\lambda \in C_{p} X$. Now $h_{C_{p} X}(\varphi)=C \eta_{X}(\varphi)=\varphi \circ \eta_{X}$, hence $h_{C_{p} X}(\varphi+\psi)=(\varphi+\psi) \circ \eta_{X}$. Thus

$$
\begin{aligned}
(\varphi+\psi) \circ \eta_{X}(x) & =(\varphi+\psi)\left(\eta_{X}(x)\right)=\varphi\left(\eta_{X}(x)\right)+\psi\left(\eta_{X}(x)\right) \\
& =h_{C_{p} X}(\varphi)(x)+h_{C_{p} X}(\psi)(x)=\left(h_{C_{p} X}(\varphi)+h_{C_{p} X}(\psi)\right)(x) .
\end{aligned}
$$

Since this holds for every $x \in X$, we have $h_{C_{p} X}(\varphi+\psi)=h_{C_{p} X}(\varphi)+h_{C_{p} X}(\psi)$.

The proof that $h_{C_{p} X}(\varphi \cdot \psi)=h_{C_{p} X}(\varphi) \cdot h_{C_{p} X}(\psi)$ is similar. We also have $h_{C_{p} X}(t \varphi)$ $=t h_{C_{p} X}(\varphi)$, where $t$ is a scalar.

Proposition 3.11. For any $f: Y \rightarrow X$, the map $C_{p} f: C_{p} X \rightarrow C_{p} Y$ preserves the ring structure. 
Proof. Since $C_{p} f$ acts by composition on the right, the result is clear. We will verify one case only: $C_{p} f(\varphi+\psi)=C_{p} f(\varphi)+C_{p} f(\psi)$. Then

$$
\begin{aligned}
C_{p} f(\varphi+\psi)(y) & =(\varphi+\psi)(f(y))=\varphi(f(y))+\psi(f(y)) \\
& =C_{p} f(\varphi)(y)+C_{p} f(\psi)(y)=\left(C_{p} f(\varphi)+C_{p} f(\psi)\right)(y) .
\end{aligned}
$$

Since the equality holds for every $y \in Y, C_{p} f(\varphi+\psi)=C_{p} f(\varphi)+C_{p} f(\psi)$.

Problem 3.12. Characterize fully the Eilenberg-Moore category of $M$-algebras.

\section{REFERENCES}

[1] J. Adámek, H. Herrlich, and G. E. Strecker, Abstract and Concrete Categories, Pure and Applied Mathematics, John Wiley \& Sons, New York, 1990.

[2] A. V. Arkhangel'skiŭ, Topological Function Spaces, Mathematics and Its Applications (Soviet Series), vol. 78, Kluwer Academic Publishers, Dordrecht, 1992.

Koena R. Nailana: Department of Mathematics, Applied Mathematics and Astronomy, UniVersity of SOUth AFricA, P.O. Box 392, Pretoria 0003, SOUTH AFricA

E-mail address: nai1akr@unisa.ac.za 


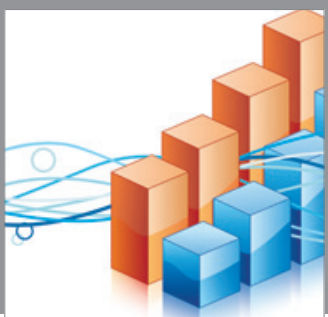

Advances in

Operations Research

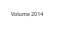

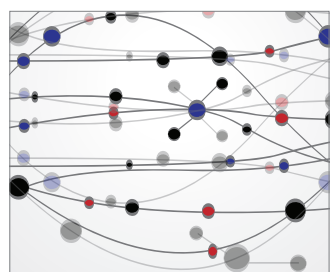

\section{The Scientific} World Journal
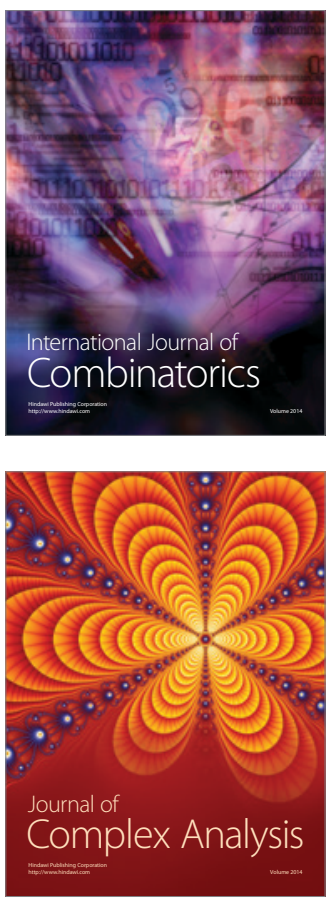

International Journal of

Mathematics and

Mathematical

Sciences
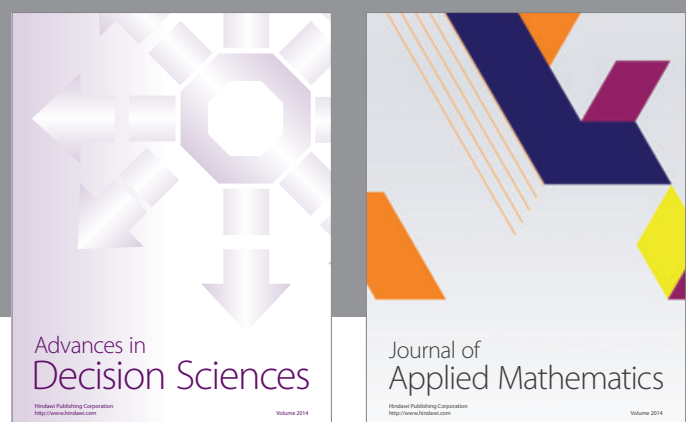

Journal of

Applied Mathematics
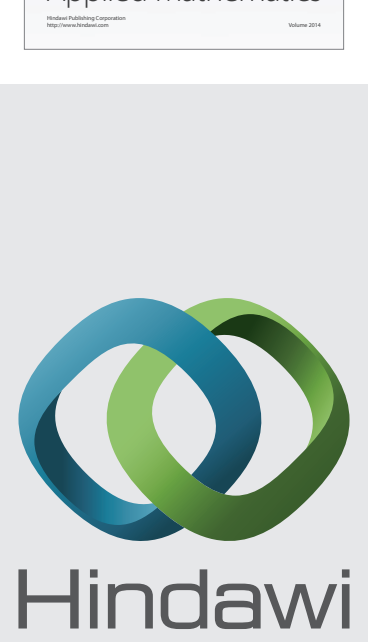

Submit your manuscripts at http://www.hindawi.com
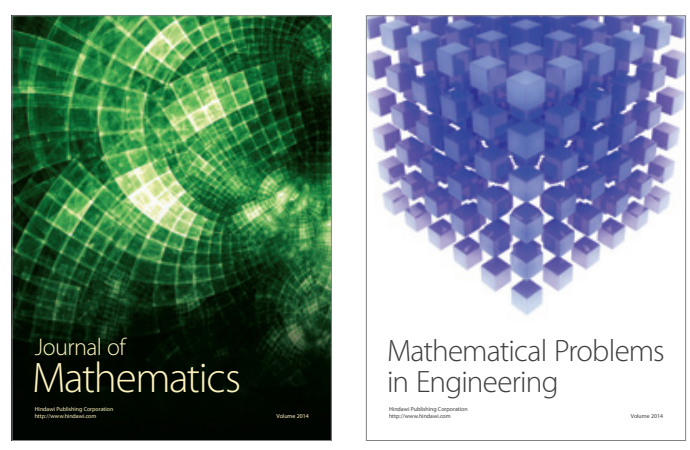

Mathematical Problems in Engineering
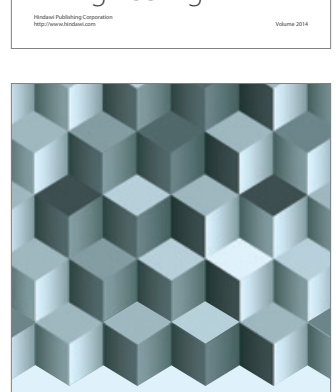

Journal of

Function Spaces
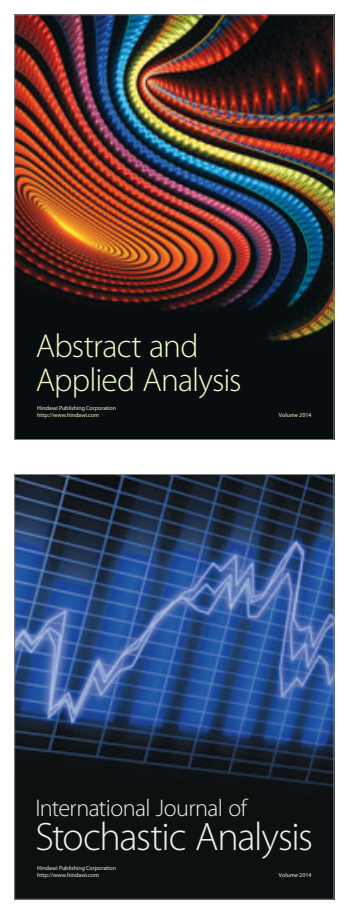

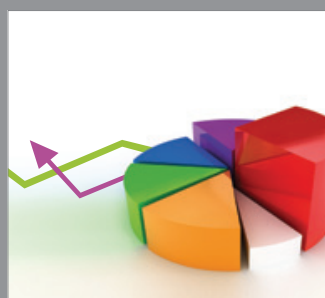

ournal of

Probability and Statistics

Promensencen
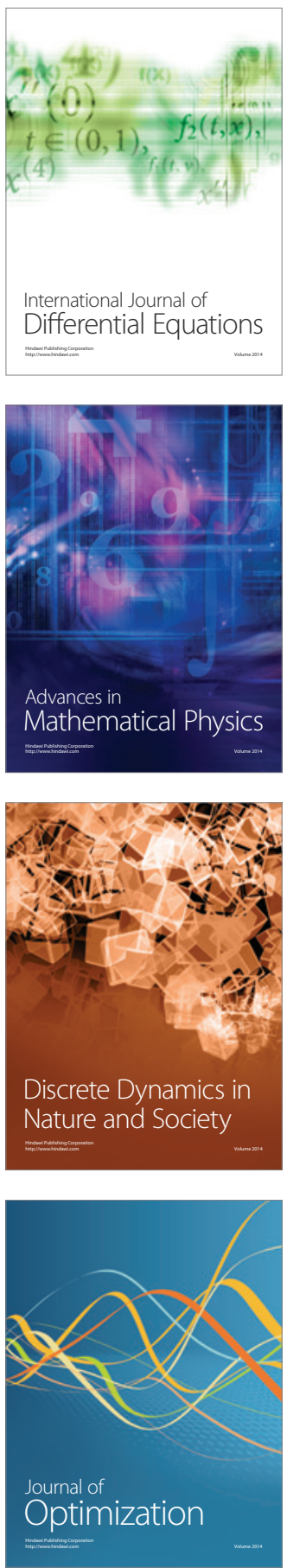